\title{
Deslocamento de Artibeus lituratus (Olfers, 1818) (Mammalia, Chiroptera) entre ilha e continente no Estado do Rio de Janeiro, Brasil
}

\author{
Luis Fernando Menezes Jr. ${ }^{1,3}$, Ana Carolina Duarte ${ }^{1}$, Roberto Leonan Morim Novaes ${ }^{1}$,
}

Ana Cristina Façanha ${ }^{1}$, Adriano Lúcio Peracchi', Luciana de Moraes Costa ${ }^{2}$,

Agata de Freitas Prata Dias e Fernandes ${ }^{2}$ \& Carlos Eduardo Lustosa Esbérard ${ }^{2}$

${ }^{1}$ Laboratório de Mastozoologia, Instituto de Biologia, Universidade Federal Rural do Rio de Janeiro - UFRRJ, Km 47 da antiga estrada Rio-São Paulo, CEP 23890-000, Seropédica, RJ, Brasil

${ }^{2}$ Laboratório de Diversidade de Mamíferos, Instituto de Biologia, Universidade Federal Rural do Rio de Janeiro - UFRRJ,

Km 47 da antiga estrada Rio-São Paulo, CP 74507, CEP 23851-970, Seropédica, Rio de Janeiro, Brasil, e-mail: lucianamcosta@yahoo.com.br

${ }^{3}$ Autor para correspondência: Luis Fernando Menezes Jr., e-mail: promorcegos@yahoo.com.br

MENEZES-Jr., L.F., DUARTE, A.C., NOVAES, R.L.M., FAÇANHA, A.C., PERACCHI, A.L., COSTA, L.M., PRATA, A.F.D. \& ESBÉRARD, C.E.L. 2008. Movement of Artibeus lituratus (Olfers, 1818) (Mammalia, Chiroptera) between island and continent on State of Rio de Janeiro, Brazil. Biota Neotrop. 8(2): http:// www.biotaneotropica.org.br/v8n2/en/abstract?short-communication+bn00808022008.

Abstract: One individual of Artibeus lituratus, marked with a colored and numbered plastic color necklace was recaptured on July $18^{\text {th }}, 2007$ at the Parque Natural Municipal da Serra do Mendanha, Rio de Janeiro, Brazil, 96 days after being captured and marked at Itacuruça Island, located on southern Rio de Janeiro State. The linear distance between the plots of marking and recapture suggests a minimum movement of $35 \mathrm{~km}$, being $500 \mathrm{~m}$ oversea. Keywords: bat, mark, recapture, Atlantic Forest.

MENEZES-Jr., L.F., DUARTE, A.C., NOVAES, R.L.M., FAÇANHA, A.C., PERACCHI, A.L., COSTA, L.M., PRATA, A.F.D. \& ESBÉRARD, C.E.L. 2008. Deslocamento de Artibeus lituratus (Olfers, 1818) (Mammalia, Chiroptera) entre ilha e continente no Estado do Rio de Janeiro, Brazil. Biota Neotrop. 8(2): http://www. biotaneotropica.org.br/v8n2/pt/abstract?short-communication+bn00808022008.

Resumo: Em 18 de julho de 2007, um indivíduo de Artibeus lituratus marcado com coleira plástica numerada foi recapturado no Parque Natural Municipal da Serra do Mendanha, município do Rio de Janeiro, 96 dias após ter sido capturado e marcado na Ilha de Itacuruça, no sul do Estado do Rio de Janeiro. A distância linear entre os pontos de marcação e recaptura sugere um deslocamento de no mínimo $35 \mathrm{~km}$, sendo $500 \mathrm{~m}$ sobre o mar.

Palavras-chave: morcego, marcação, recaptura, Mata Atlântica. 


\section{Introdução}

Grandes deslocamentos são conhecidos entre morcegos frugívoros sobre áreas urbanas (Esbérard 2003) e florestais (Bernard \& Fenton 2003), tornando estes estudos importantes para a compreensão de diversos aspectos ecológicos, como a busca por recursos alimentares e por abrigos (Heithaus \& Fleming 1978, Fleming \& Heithaus 1986, Fleming 1991). Sendo assim, a mobilidade tem importância essencial para a sobrevivência de algumas espécies (Kozakiewicz \& Szacki 1995, Yabe \& Marques 2001). Esses padrões de movimentação são determinados pela estrutura do hábitat, como também por padrões de comportamento de cada espécie (Graham 2001), e é particularmente notável em grupos de morcegos (Bernard \& Fenton 2003, Bianconi et al. 2006, Albrecht et al. 2007).

Grandes deslocamentos de morcegos já foram reportados também por Bernard \& Fenton (2003) que constataram movimentação de 14 espécies entre abrigos e sobre fragmentos florestais na Amazônia. Neste estudo, espécies do gênero Artibeus Leach, 1821 demonstraram grande capacidade de movimentação, explorando diversos fragmentos. Segundo Bianconi et al. (2006), em fragmentos florestais do Paraná, espécies do gênero Artibeus exibiram uma baixa freqüência de recaptura em uma mesma área, sugerindo alta mobilidade e grande área de forrageio, provavelmente relacionada à exploração na busca por recursos, em especial Ficus L. (Moraceae), seu alimento preferencial. Em contraste, segundo estes mesmos autores, Carollia perspicillata (Linnaeus, 1758) apresentou uma área restrita de forrageio, determinada provavelmente pela abundância de Piper L. (Piperaceae), planta reconhecida como seu alimento preferencial.

Costa et al. (2006) reportaram deslocamento de Artibeus fimbriatus Gray, 1838 por cerca de $21,7 \mathrm{~km}$, sendo $17 \mathrm{~km}$ sobre o mar e $4,7 \mathrm{~km}$ sobre terra, sugerindo que a espécie, e provavelmente as demais do mesmo gênero, mantém capacidade de deslocamento entre ilhas e áreas continentais, o que sugere a existência de fluxo gênico entre os indivíduos presentes nestas áreas. Albrecht et al. (2007) analisaram a mobilidade em duas pequenas espécies de morcegos filostomídeos, Micronycteris microtis Miller, 1898 e Artibeus watsoni Thomas, 1901. A primeira demonstrou um comportamento sedentário, não cruzando água, enquanto $A$. watsoni demonstrou deslocar-se até 180 metros sobre água, o que sugere que esta espécie, por ser frugívora, acaba por explorar diversos habitats na busca por recursos alimentares.

O objetivo deste trabalho é relatar o deslocamento do morcego frugívoro Artibeus lituratus entre ilha e continente no Estado do Rio de Janeiro.

\section{Material e Métodos}

$\mathrm{O}$ indivíduo de $A$. lituratus em questão foi marcado na Ilha de Itacuruça (22 $56^{\circ} 11.20^{\prime \prime} \mathrm{S}$ e $43^{\circ} 53^{\prime} 04.37^{\prime}$ 'W), sul do Estado do Rio de Janeiro, que dista cerca de 500 metros do continente e tem uma área de 994 hectares com grandes fragmentos de Mata Atlântica em estágio secundário e com grande influência antrópica. Já a recaptura do morcego, foi feita no Parque Natural Municipal (PARNA)

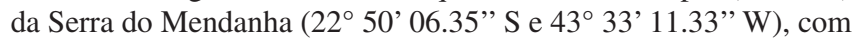
1450 ha., localizado na serra do Mendanha e do Quitumbo, região que abriga um dos últimos remanescentes de Mata Atlântica bem preservada em estágios primário e secundário do Município do Rio de Janeiro. A região está sendo amostrada desde 2005 em intervalos irregulares, já tendo sido realizadas 16 coletas e analisadas mais de 250 capturas e recapturas.

As capturas foram feitas com o uso de redes de neblina de $7 \times 2,5 \mathrm{~m}$ que foram armadas em trilhas, clareiras e cursos de água imediatamente após o anoitecer e retiradas nas primeiras horas da manhã. Até o momento, foi totalizado um esforço amostral de $13440 \mathrm{~m}^{2} /$ hora, conforme Straube \& Bianconi (2002). Os morcegos capturados foram acondicionados em sacos de pano branco, pesados e foram feitas medições do antebraço, cabeça e tíbia, e quando possível, a identificação da espécie foi feita no próprio local. Os morcegos da Ilha de Itacuruça e do PARNA da Serra do Mendanha foram marcados com método modificado de Esbérard \& Daemon (1999), sendo usado um cilindro plástico de cor diferente para cada região amostrada, a fim de facilitar a identificação da origem do animal.

\section{Resultados e Discussão}

Em 18 de julho de 2007, às 22 horas 10 minutos, uma fêmea de Artibeus lituratus em condição não reprodutiva, com comprimento de antebraço 71,0 mm e peso de 70 gramas foi recapturado no PARNA da Serra do Mendanha, usando coleira plástica numerada, após ter sido capturado e marcado no dia 12 de abril de 2007 na Ilha de Itacuruça. O resultado revela uma distância de $34,79 \mathrm{~km}$ entre os pontos de marcação e recaptura, o que sugere que o morcego tenha se deslocado no mínimo $500 \mathrm{~m}$ sobre o mar e 34,29 km sobre o continente.

Alguns autores sugerem que a movimentação de morcegos esteja relacionada à disponibilidade de recursos e hábitat, especialmente comida e abrigo (Heithaus \& Fleming 1978, Fleming \& Heithaus 1986, Fleming 1991). Desta forma, a fragmentação florestal acaba por reduzir a disponibilidade e modificar a distribuição destes recursos na paisagem, o que pode levar os morcegos a explorarem outras áreas (Estrada \& Coates-Estrada 2002, Evelyn \& Stiles 2003) e até mesmo migrarem longas distâncias, explorando ilhas costeiras e áreas continentais (Costa et al. 2006).

O fato aqui reportado acrescenta mais um registro do deslocamento de longas distâncias feito por algumas espécies de morcegos. Sendo assim, o resultado corrobora com os demais trabalhos publicados sobre deslocamento de morcegos frugívoros, especialmente do gênero Artibeus (Bernard \& Fenton 2003, Bianconi et al. 2006, Costa et al. 2006, Albrecht et al. 2007), e sugere que diversas espécies do gênero Artibeus mantêm fluxo gênico entre diferentes fragmentos.

\section{Agradecimentos}

À Secretaria Municipal de Meio Ambiente e Conservação do Rio de Janeiro pela autorização de pesquisa no PARNA da Serra do Mendanha. Ao Sr. Jairo e esposa, proprietários do sítio onde foi feita a recaptura. À Carolinne I. Dias Gomes pela ajuda na tradução do abstract.

\section{Referências Bibliográficas}

ALBRECHT, L., MEYER, C.F.J. \& KALKO, E.K.V. 2007. Differential mobility in two small phyllostomid bats, Artibeus watsoni and Micronycteris microtis, in a fragmented neotropical landscape. Acta Theriol. 52(2): 141-149.

BERNARD, E. \& FENTON, M.B. 2003. Bat mobility and Roosts in a Fragmented Landscape in Central Amazonia, Brazil. Biotropica 35(2): 262-277.

BIANCONI, G.V., MIKICH, S.B. \& PEDRO, W.A. 2006. Movements of bats (Mammalia, Chiroptera) in Atlantic Forest remnants in southern Brazil. Rev. Bras. de Zool. 23(4): 1199-1206.

COSTA, L.M., PRATA, A.F.D., MORAES, D., CONDE, C.F.V., JORDÃONOGUEIRA, T. \& ESBÉRARD, C. E. L. 2006. Deslocamento de Artibeus fimbriatus sobre o mar. Chiropt. Neotrop.12(2): 289-290.

ESBÉRARD, C.E.L. 2003. Marcação e deslocamento em morcegos. Divulgação do Museu de Ciências e Tecnologia 2: 23-24.

ESBÉRARD, C.E.L. \& DAEMON, C. 1999. Um novo método para marcação de morcegos. Chiropt. Neotrop. 05 (1-2): 116-117.

ESTRADA, A. \& COATES-ESTRADA, R. 2002. Bats in continuos forest, forest fragments and in a agricultural mosaic habitat-island at Los Tuxtlas, Mexico. Biol. Conserv. 103(2): 237-245. 
EVELYN, M.J. \& STILES, D.A. 2003. Roosting requirements of two frugivorous bats (Sturnira lilium and Artibeus intermedius) in fragmented neotropical forest. Biotropica 35(3): 405-418.

GRAHAM, C.H. 2001. Factors influencing movement patterns of Kell-billed Toucans in a fragmented tropical landscape in Southern Mexico. Conserv. Biol. 15(6): 1789-1798.

FLEMING, T.H. 1991. The relationship between body size, diet, and habitat use in frugivorous bats, genus Carollia (Phyllostomatidae). J. Mammal. 72(3): 493-501.

FLEMING, T.H. \& HEITHAUS, E.R. 1986. Seasonal foranging behavior of the frugivorous bat Carollia perspicillata. J. Mammal. 67(4): 660-671.

HEITHAUS, E.R. \& FLEMING, T.H. 1978. Foraging movements of a frugivorous bat, Carollia perspicillata (Phyllostomidae). Ecol. Monogr. 48(2): 127-143.
KOZAKIEWICZ, M. \& SZACKI, J. 1995. Movements of small mammals in a landscape: patch restriction or nomadism? p. 78-94. In Landscape approaches in mammalian ecology and conservation. (LIDICKER-JR, W.Z. Ed). University of Minnesota Press, Minneapolis. 215 p.

STRAUBE, F.C. \& BIANCONI, G.V. 2002. Sobre a grandeza e a unidade utilizada para estimar esforço de captura com utilização de redes-deneblina. Chirop. Neotrop. 08(1-2): 150-152.

YABE, R.S. \& MARQUES, E.J. 2001. Deslocamento de aves entre capões no Pantanal Mato-grossense e sua relação com a dieta, pp. 103-123. In Ornitologia e conservação: da ciência às estratégias (ALBUQUEQUE, J.L.B., CÂNDIDO-JR, J.F., STRAUBE, F.C. \& ROOS, A.L. Eds). Editora Unisul, Tubarão. 341p.

Recebido em 14/10/07

Versão Reformulada recebida em 17/03/08

Publicado em 12/04/08 